\title{
Análise da variabilidade da frequência cardíaca em idosos na unidade de terapia intensiva submetidos à manobra de Bag Squeezing
}

Analysis of heart rate variability in elderly in the intensive care unit submitted to the Bag Squeezing maneuver

Análisis de la variabilidad de la frecuencia cardíaca en ancianos de la unidad de cuidados intensivos sometidas a la maniobra de Bag Squeezing

\section{Resumo}

Objetivo: analisar o efeito da manobra de Bag squeezing na variabilidade cardíaca de idosos internados em unidade de terapia intensiva no Hospital Regional Norte no município de Sobral-CE. Método: Tratou-se de um estudo intervencionista de abordagem quantitativa, realizado na Unidade de Terapia Intensiva do Hospital Regional Norte no Município de Sobral, Ceará, no período de Janeiro de 2015 a Março de 2015. A pesquisa contemplou a população idosa internada na unidade de terapia intensiva no município de Sobral, e realizou-se a manobra de Bag squeezing, mensurando-se o impacto da mesma sobre a variabilidade da frequência cardíaca dos participantes. Resultados: estiveram envolvidos 20 sujeitos, independentemente do sexo, com média de idade de 72 anos, tendo como patogenia mais recorrente a pneumonia. Após a manobra, observou-se diminuição dos sinais vitais e melhora na saturação de oxigênio. Não houve diferença significativa quanto à modulação autonômica cardíaca. Conclusão: Conclui-se que a 
manobra de Bag squeezing é uma técnica que não causa repercussões na variabilidade da frequência cardíaca sendo de grande efeito na saturação de oxigênio, redução das frequências cardíaca e respiratória, além da pressão arterial.

Palavras-chave: Unidade de Terapia Intensiva; Terapia respiratória/métodos; Modalidades de fisioterapia.

\begin{abstract}
Aim: To analyze the effect of Bag squeezing maneuver in heart rate variability in elderly patients admitted to an intensive care unit in the Northern Regional Hospital in the city of Sobral-CE. Method: It was an interventional study with a quantitative approach, carried out in the Intensive Care Unit of Hospital Regional Norte in the municipality of Sobral, Ceará, from January 2015 to March 2015. The research covered the elderly population hospitalized in the therapy unit intensive in the municipality of Sobral, and it performed the Bag squeezing maneuver, measuring its impact on the participants' heart rate variability. Results: We selected 20 subjects, regardless of gender, with a mean age of 72 years, with the most frequent pathogenic pneumonia, which showed a decrease in vital signs after the effect of the maneuver during the search. Regarding maneuver does not influence significantly the autonomic modulation, we can see a reduction of RR complex during operation and maintenance for an extended time. However, this is not noticeable in SDNN index during the maneuver, featuring a minor change in heart rate variability. Conclusion: We conclude that the Bag squeezing maneuver is a technique that does not cause repercussions on heart rate variability and has a significant effect on oxygen saturation, reduction of heart and respiratory rates, besides blood pressure.
\end{abstract}

Keywords: Intensive Care Unit; Respiratory therapy/ methods; Physical therapy modalities.

\title{
Resumen
}

Objetivo: analizar el efecto de la maniobra Bag squeezing sobre la variabilidad cardíaca en pacientes ancianos ingresados en una unidad de cuidados intensivos del Hospital Regional Norte del municipio de Sobral-CE. Método: Se trata de un estudio intervencionista con abordaje cuantitativo, realizado en la Unidad de Cuidados Intensivos del Hospital Regional Norte del municipio de Sobral, Ceará, de enero de 2015 a marzo de 2015. La investigación incluyó a la población anciana hospitalizada en la unidad de cuidados intensivos. en el municipio de Sobral, y se realizó la maniobra Bag squeezing, midiendo su impacto en la variabilidad de la frecuencia cardíaca de los participantes. Resultados: participaron 20 sujetos, independientemente del sexo, con una edad media de 72 años, siendo la neumonía la patogenia más recurrente. Tras la maniobra, hubo una disminución de los signos vitales y una mejoría de la saturación de oxígeno. No hubo diferencias significativas con respecto a la modulación autónoma cardíaca. Conclusión: Se concluye que la maniobra Bag squeezing es una técnica que no repercute en la variabilidad de la frecuencia cardíaca y tiene un gran efecto en la saturación de oxígeno, reducción de la frecuencia cardíaca y respiratoria, además de la presión arterial.

Palabras clave: Unidad de Cuidados Intensivos; Terapia / métodos respiratorios; Modalidades de fisioterapia.

\section{Introdução}

O processo de envelhecimento gera inúmeras alterações fisiológicas, que culminam na maior vulnerabilidade ao desenvolvimento de morbidades em idosos fazendo com que estes necessitem de maior suporte hospitalar por doenças crônicas degenerativas (Rossetto et al., 2019). A Unidade de Terapia Intensiva (UTI) é um dos setores que mais sofre com o fenômeno do crescimento do número de idosos, pois esses pacientes são mais susceptíveis a apresentar problemas crônicos de saúde cuja agudização exacerba doenças que exigem complicados tratamentos, ocupando assim cada vez mais leitos destas unidades (Gomes \& Gomes, 2012).

A UTI é um ambiente hospitalar que visa a manutenção da vida e a recuperação da saúde das pessoas que se encontram em estado crítico. Para tal, dispõe de uma série de recursos tecnológicos, aparelhos de monitorização, exames laboratoriais frequentes e a avaliação clínica constante, visando a manutenção da vida (Pina et al., 2009). A UTI é um dos setores que mais sofre com o fenômeno do crescimento do número de idosos, pois esses pacientes são mais susceptíveis a apresentar problemas crônicos de saúde cuja agudização exacerba doenças que exigem tratamentos complexos, ocupando assim cada vez mais leitos destas unidades (Gomes \& Gomes, 2012). Todavia, a UTI apesar de ser um excelente local de recuperação aos pacientes críticos, promove inúmeros danos, em especial aos pacientes sob ventilação mecânica os quais, uma vez sedados, entram em quadro de imobilismo que conduz a redução da atividade mucociliar, consequentemente, levando ao acumulo de secreções.

Para adequada assistência dos indivíduos em uma UTI a fisioterapia apresenta um arsenal de técnicas e manobras eficazes no tratamento desses pacientes, objetivando otimizar a complacência do sistema respiratório, promover a higiene 
brônquica e melhorar a ventilação pulmonar (Lobo et al., 2010). Em meio às manobras de remoção de secreção brônquica e expansão pulmonar utilizadas em pacientes submetidos ao uso na Ventilação Mecânica invasiva (VMI) pode-se citar a hipersinsuflação, a qual pode ser realizada manualmente utilizando uma bolsa ventilatória ou por meio do ventilador mecânico (Paschoal et al., 2006).

A hiperinsuflação manual ou bag squeezing visa melhorar a ventilação alveolar, mobilizar secreções brônquicas, reverter áreas atelectasiadas, além de reestabelecer a complacência estática. No entanto, por fazer uso da pressão positiva, pode acarretar em alterações hemodinâmicas, em especial sobre o sistema cardiovascular com a redução da pos-carga e debito cardíaco, que leva a um estado compensatório com o aumento da frequência cardíaca (Lobo et al., 2010).

Devido ao elevado número de idosos internados em UTIs e à escassez de estudos que abordem a repercussão da manobra de Bag squeezing na variabilidade da frequência cardíaca (VFC) deste público, buscou-se entender suas alterações fisiológicas no organismo de cada indivíduo sendo esta pesquisa um diferencial acadêmico e fisioterapêutico, agregando conhecimentos. Para tanto, objetivou-se, investigar o efeito da manobra de Bag squeezing na variabilidade cardíaca dos idosos internados em unidade de terapia intensiva em um Hospital de referência do município de Sobral-CE.

\section{Metodologia}

Trata-se de um estudo intervencionista de abordagem quantitativa, realizado na UTI de um Hospital de referência do Município de Sobral, no período de janeiro a março de 2015. A população foi composta por idosos internados na UTI do referido hospital e a amostra constituída por todos os que possuíssem viabilidade e critérios para execução da manobra e avaliações escolhidas. A pesquisa seguiu os princípios éticos da resolução 466/12 do Conselho Nacional de Saúde (CNS) (Brasil, 2013; Novoa \& Novoa, 2014), a qual foi assinada um termo de assentimento pelos responsáveis dos voluntários, bem como a pesquisa foi aprovada pelo Comitê de Ética e Pesquisa da Escola de Saúde Pública do Ceará - ESP/ CE sob o Número do Parecer: 889.635.

Foram incluídos indivíduos com faixa etária entre 60 e 90 anos, independentemente do gênero, que estavam internados, no mínimo, há três dias na UTI fazendo uso de ventilação mecânica invasiva, que tivessem indicação da técnica, e que os responsáveis concordassem que participassem da pesquisa por meio da assinatura do termo de assentimento livre e esclarecido (TALE). Foram excluídos os que apresentaram instabilidade hemodinâmica antes da manobra, lesões torácicas, cirurgias toracoabdominal e, os responsáveis solicitarem a retirada da pesquisa.

Para registro dos dados foi preenchida uma ficha contendo as seguintes informações: idade, patogenia, medicação, sinais vitais e dados da VFC (antes, durante, após 10 e 30 minutos).

Previamente à coleta, agendou-se com o responsável pela UTI uma data para seleção dos pacientes e execução do estudo. Na oportunidade foi explanada a pesquisa aos responsáveis pelo paciente e, em seguida, foi realizado o convite para a participação, e neste momento foi feito a leitura e a assinatura do TALE. Os fisioterapeutas responsáveis receberam treinamento e capacitação para utilização da manobra e do frequencímetro, além da avaliação, da execução e da interpretação dos dados.

O frequencímetro foi afixado por meio de uma cinta/faixa elástica regulável na altura do peito esquerdo do paciente. O frequencímetro é um aparelho de precisão composto por uma cinta (polar), instalada na altura do peito do paciente, e um monitor. Este aparelho tem como função medir e controlar os batimentos cardíacos de uma pessoa (Mata et al., 2012). O monitoramento dos batimentos cardíacos é realizado por meio do envio da frequência cardíaca a um monitor específico, que é salvo em memória de computador e analisado por meio de um software (Protainer ${ }^{\circledR}$ ) e devidamente acompanhado pelos profissionais da área da saúde. 
A coleta da VFC ocorreu em quatro etapas conforme descrito a seguir: 1) realizada antes da execução da manobra de bag squeezing, com o paciente em repouso por 5 minutos sem intervenção da equipe; 2) com duração de 5 minutos, ocorreu durante a execução da manobra; 3) após 10 minutos da realização da manobra; 4) após 30 minutos da realização da manobra (nas etapas 3 e 4 o paciente permaneceu em repouso e sem intervenções).

A análise da VFC, por meio de métodos lineares, pode ser realizada de acordo com dois parâmetros: tempo e domínio da frequência, conforme recomendado por Cambri et al. (2008) e Cambri et al., (2008). Todos esses dados da VFC foram analisados por meio do programa Kubios HRV Analysis® (versão 2.2), que emite um relatório contendo os valores de todos os dados citados e, portanto, foram inseridos em um banco de dados para posterior aplicação nos testes estatísticos.

A técnica de Bag squeezing é realizada por dois fisioterapeutas, consistindo na aplicação de uma série de incursões respiratórias amplas e profundas, com uma pausa inspiratória de 3 segundos, seguidos de rápidas expirações para simular a tosse e liberação repentina com AMBU, gerando um alto fluxo expiratório (Azeredo, 2002). O protocolo teve a duração de 5 minutos, seguido de aspiração endotraqueal por sistema aberto, com sonda de aspiração traqueal.

Os dados foram tratados seguindo as normas do Task Force e, em seguida, digitados em um banco de dados utilizando o programa Epi Info (versão 6.04d, Centers for Disease Control and Prevention, EUA), consequentemente, analisados pelo software SPSS (versão 21.0)

A análise inicial foi realizada por meio de uma estatística descritiva, incluindo tabulações de acordo com as variáveis selecionadas e para o cálculo inferencial verificando a normalidade dos dados, distribuição da frequência simples das variáveis de interesse desse estudo. Em seguida, realizou-se uma análise estatística por meio do SPSS, na qual ao inferir o teste de Kolmogorov-Smirnov, detectou-se normalidade para todas as variáveis do estudo, sendo, portanto, utilizados testes paramétricos, como o teste $\mathrm{t}$ de Student para amostras pareadas e ANOVA com post hoc Bonferroni. Considerou-se significância estatística quando $\mathrm{p} \leq 0,05$, sendo os dados expressos em tabelas.

\section{Resultados}

Participaram do estudo 20 sujeitos internados na UTI de um hospital público de referência na região noroeste do Estado do Ceará, sendo que destes $65 \%$ era do gênero masculino $(n=13)$ com média de idade de 72 anos $(60-86 ; \pm 8,9)$. Todos os sujeitos faziam uso de VMI nas modalidades assisto-controlada e pressão positiva contínua na via aérea associada à pressão de suporte (CPAP com PSV), os quais recebiam em média de 33\% de fração de oxigênio $\left(\mathrm{FIO}_{2}\right)$, variando de 21 a $60 \%$ $( \pm 11,65)$ e $75 \%(n=15)$ fazia uso de tubo orotraqueal (TOT), enquanto que $25 \%(n=5)$ utilizava o traqueóstomo (TQT).

Quanto às patogenias, as mais recorrentes neste grupo foram: pneumonia $(45 \%, \mathrm{n}=9)$, seguida de insuficiência respiratória aguda (IRA), apendicite e choque séptico, cada um com 10\% (n=2), conforme Tabela 1. 
Tabela 1: Patogenias mais recorrentes dos idosos admitidos na pesquisa, Sobral, 2015.

\begin{tabular}{lcc}
\hline \multicolumn{1}{c}{ Patogenia } & Frequência & \\
& Absoluta (n) & Relativa (\%) \\
\hline Pneumonia & 9 & 45 \\
Insuficiência Respiratória Aguda & 2 & 10 \\
Apendicite & 2 & 10 \\
Choque Séptico & 2 & 10 \\
Hepatite & 1 & 5 \\
Abcesso Pulmonar & 1 & 5 \\
Hemorragia Digestiva & 1 & 5 \\
Encefalopatia Metabólica & 1 & 5 \\
Doença Pulmonar Obstrutiva Crônica & 1 & 5 \\
\hline Total & $\mathbf{2 0}$ & $\mathbf{1 0 0}$ \\
\hline
\end{tabular}

Fonte: Dados da pesquisa.

Ao realizar a inferência dos dados de frequência cardíaca $(\mathrm{FC})$ e respiratória $(\mathrm{FR})$, saturação de oxigênio $\left(\mathrm{SatO}_{2}\right)$, pressão arterial sistólica (PAS) e diastólica (PAD), antes e após a aplicação da manobra de Bag squeezing , percebeu-se diferença estatistíca, por meio do teste t para amostras pareadas, conforme apresentado na Tabela 2.

Tabela 2: Análise comparativa dos sinais vitais, antes e após à Manobra de Bag squeezing, em idosos de uma Unidade de Terapia Intensiva de Sobral, 2015.

\begin{tabular}{|c|c|c|c|c|c|c|}
\hline Sinais Vitais & & Média & $\begin{array}{c}\text { Desvio } \\
\text { Padrão } \\
( \pm)\end{array}$ & $\begin{array}{c}\text { Erro do } \\
\text { Padrão da } \\
\text { Média }\end{array}$ & IC (95\%) & Valor $\mathbf{p}$ \\
\hline \multirow{2}{*}{ Frequência Cardíaca (bpm) } & Antes & 83,8 & 16,8 & 3,7 & \multirow{2}{*}{$(0,6 / 7,3)$} & \multirow{2}{*}{$0,0001^{*}$} \\
\hline & Depois & 80,4 & 15,4 & 3,4 & & \\
\hline \multirow{2}{*}{ Frequência Respiratória (ipm) } & Antes & 20 & 4,7 & 1,1 & \multirow{2}{*}{$(0,3 / 1,7)$} & \multirow{2}{*}{$0,0001 *$} \\
\hline & Depois & 18 & 4,3 & 0,9 & & \\
\hline \multirow{2}{*}{ Saturação de Oxigênio (\%) } & Antes & 96 & 2,8 & 0,6 & \multirow{2}{*}{$(-2,0 /-0,3)$} & \multirow{2}{*}{$0,0001 *$} \\
\hline & Depois & 99 & 2,2 & 0,5 & & \\
\hline \multirow{2}{*}{ Pressão Arterial Sistólica (mmHg) } & Antes & 131 & 21,8 & 4,8 & \multirow{2}{*}{$(5,9 / 19,6)$} & \multirow{2}{*}{$0,0001^{*}$} \\
\hline & Depois & 117 & 26,2 & 5,8 & & \\
\hline \multirow{2}{*}{ Pressão Arterial Diastólica (mmHg) } & Antes & 69 & 17,1 & 3,8 & \multirow{2}{*}{$(5,2 / 8,2)$} & \multirow{2}{*}{$0,012 *$} \\
\hline & Depois & 66 & 11,9 & 2,6 & & \\
\hline
\end{tabular}

Legenda: $\mathrm{IC}=$ Intervalo de Confiança. Utilizado teste $\mathrm{t}$ para amostras pareadas com $\mathrm{p} \leq 0,05$. *Significância estatística. Fonte: dados da pesquisa.

Pôde-se observar uma predominância do sistema nervoso autônomo parassimpático imediatamente após à aplicação da técnica, uma vez que houve redução nos valores das FC, FR, PAS e PAD, sendo considerada melhora e estabilização dos dados. Apesar da $\mathrm{SatO}_{2}$ ter apresentado um aumento de seus valores, isto não se justificaria pela ação do sistema parassimpático, já que sua ação seria de depressão do nível de oxigenação em detrimento da redução do volume de ejeção (perfusão) e da ventilação por causa da FR, mas, em decorrência da fase de aspiração de secreção promovida pela própria manobra.

Para análise da VFC, domínio tempo, nos momentos avaliados, utilizou-se o teste ANOVA com nível de significância de $5 \%$, pôde-se perceber que a manobra não influencia significativamente na modulação autonômica. Entretanto, tal fato não é 
perceptível no índice SDNN durante a execução da manobra, caracterizando uma pequena alteração na variabilidade da frequência cardíaca. Este índice não permite a identificação de qual sistema está alterado (simpático ou parassimpático).

Quando analisados os índices pNN50 e rMSSD pôde-se observar uma redução contínua e mantida durante todas as fases da análise, mostrando que a manobra influência na ativação do sistema simpático, visivelmente em longo prazo, conforme Tabela 3.

Tabela 3: Análise da variabilidade da frequência cardíaca (domínio tempo), antes, durante, após 10 e 30 minutos da Manobra de Bag squeezing em idosos de uma UTI de Sobral, 2015.

\begin{tabular}{lccccc}
\hline $\begin{array}{c}\text { Variável da } \\
\text { VFC }\end{array}$ & \multicolumn{4}{c}{ Média (Dp) } & Valor p* \\
\hline & Antes & $\begin{array}{c}\text { Durante a } \\
\text { Manobra }\end{array}$ & Após 10 minutos & Após 30 minutos \\
Complexo RR & $737,9( \pm 167,1)$ & $686,5( \pm 138,6)$ & $693,7( \pm 154,1)$ & $688,7( \pm 153,5)$ & $0,683^{* *}$ \\
SDNN & $41,3( \pm 21,1)$ & $46,0( \pm 22,1)$ & $34,0( \pm 19,3)$ & $39,6( \pm 20,6)$ & $0,344 * *$ \\
pNN50 & $20,8( \pm 18,7)$ & $15,9( \pm 18,7)$ & $14,8( \pm 17,4)$ & $14,7( \pm 18,2)$ & $0,682^{* *}$ \\
rMSSD & $43,9( \pm 29,6)$ & $41,4( \pm 29,4)$ & $34,5( \pm 26,0)$ & $33,5( \pm 27,6)$ & $0,582^{* *}$ \\
\hline
\end{tabular}

Legenda: VFC: Variabilidade da Frequência Cardíaca; Dp: Desvio Padrão ( \pm ); SDNN: desvio padrão de todos os intervalos RR normais gravados em um intervalo de tempo, expresso em ms; pNN50: representa a porcentagem dos intervalos RR adjacentes com diferença de duração maior que 50ms; rMSSD: é a raiz quadrada da média do quadrado das diferenças entre os intervalos RR normais adjacentes, em um intervalo, expresso em ms. ** Não houve diferença estatística.

Fonte: Dados da pesquisa.

Quando considerado o domínio frequência da VFC, a comparação dos momentos avaliados por meio do teste ANOVA revelou não haver diferença estatística significante. Ao realizar uma análise qualitativa espectral dos parâmetros da VFC, observou-se que o componente de alta frequência (high frequency - HF) sofreu influência durante a manobra, havendo um controle nos primeiros 10 minutos, enquanto que o componente de baixa frequência (low frequency - LF), obteve-se uma redução de seu valor na fase durante a manobra e após 30 minutos. Quando analisada a relação LF/HF identificou-se um aumento considerável quando comparado todas as fases com o período antes, porém não houve diferença significativa (Tabela 4). Tal resultado indica uma diminuição da ação vagal e um aumento da atividade simpática nos primeiros 10 minutos após a manobra.

Tabela 4: Análise da variabilidade da frequência cardíaca (domínio frequência), antes, durante, após 10 e 30 minutos da Manobra de Bag squeezing em idosos de uma UTI de Sobral, 2015.

\begin{tabular}{|c|c|c|c|c|c|}
\hline \multirow[t]{2}{*}{$\begin{array}{c}\text { Variável da } \\
\text { VFC } \\
\end{array}$} & \multicolumn{4}{|c|}{ Média (Dp) } & \multirow[t]{2}{*}{ Valor $\mathbf{p}^{*}$} \\
\hline & Antes & $\begin{array}{l}\text { Durante a } \\
\text { Manobra }\end{array}$ & Após 10 minutos & Após 30 minutos & \\
\hline HF & $39,6( \pm 28,1)$ & $53,8( \pm 26,0)$ & $49,4( \pm 32,2)$ & $55,7( \pm 32,1)$ & $0,070^{* *}$ \\
\hline $\mathbf{L F}$ & $19,7( \pm 10,1)$ & $1,4( \pm 1,7)$ & $24,0( \pm 10,3)$ & $1,4( \pm 1,1)$ & $0,964 * *$ \\
\hline VLF & $40,4( \pm 32,7)$ & $25,2( \pm 18,1)$ & $29,0( \pm 19,8)$ & $22,2( \pm 19,2)$ & $0,070^{* *}$ \\
\hline Relação LF/HF & $1,0( \pm 1,3)$ & $20,9( \pm 11,9)$ & $21,3( \pm 15,3)$ & $22,0( \pm 17,7)$ & $0,413 * *$ \\
\hline
\end{tabular}

Legenda: VFC: Variabilidade da Frequência Cardíaca; Dp: Desvio Padrão ( \pm ); HF: alta frquência; LF: baixa frequência; LF/HF: relação entre baixa frequência e alta frequência. *Teste estatístico utilizado: ANOVA com post hoc Bonferroni. ** Não houve diferença estatística.

Fonte: dados da pesquisa. 


\section{Discussão}

O envelhecimento, segundo Meneses et al., (2013) e Gouveia (2018), é um "processo dinâmico e progressivo, no qual há alterações morfológicas, funcionais e bioquímicas, que vão alterando progressivamente o organismo, tornando-o mais suscetível às agressões intrínsecas e extrínsecas". Os pacientes idosos são responsáveis de $42 \%$ a $52 \%$ das admissões em UTI e consomem cerca de 60\% dos leitos disponíveis. Dentre as principais causas definidas de mortalidade entre idosos brasileiros estão as doenças do trato respiratório, o que representa cerca de 60\% do total de óbitos nessa população (Kanso et al., 2013).

No presente estudo, verificou-se que a pneumonia foi a patogenia mais recorrente entre os avaliados, o que corrobora com diversos estudos que apontam que, apesar de todos avanços na assistência clínica e no tratamento antimicrobiano, a pneumonia continua sendo uma das principais causas de mortalidade em todo o mundo, especialmente em idosos (Akpınar et al., 2019; Macedo et al., 2007). Carvalho (2014) afirma que, no Brasil, os idosos são o público com maior coeficiente de mortalidade relacionado às doenças do aparelho circulatório, respiratório e neoplasias.

A bag squeezing ou Peep Zeep tem como objetivo aumentar a ventilação alveolar, utilizando a hiperinsuflação pulmonar pelo incremento da PEEP, a qual é aumentada para $15 \mathrm{cmH} 2 \mathrm{O}$ e após cinco ciclos ventilatórios, a PEEP é reduzida a zero, acompanhada de vibro-compressão torácica manual. Esta manobra promove a expansão dos alvéolos colapsados, aumenta o fluxo aéreo para as regiões atelectasiadas por meio dos canais colaterais e da renovação de surfactante nos alvéolos (Andrade, 2018). No entanto, resulta em algumas alterações hemodinâmicas, com potenciais efeitos sobre o sistema cardiovascular e pressões intrapleurais.

Berti et al., (2012) realizaram um estudo com hiperinsuflação manual combinada a compressão torárica expiratória e percebeu que a técnica corroborou para acelerar o processo de melhora do quadro, além de reduzir a extensão da lesão pulmonar e o tempo de permanência na UTI (Berti et al., 2012), esta pesquisa, apontou um aumento da saturação periférica de oxigênio, confirmando os benefícios da técnica para melhor funcionalidade do sistema respiratório. Em contrapartida, observou-se a redução da frequência cardíaca e da pressão arterial após a realização da técnica.

Sabe-se que a FC é regulada pelo Sistema Nervoso Autônomo (SNA). Os barorreceptores, células nervosas especializadas localizadas no arco aórtico e no seio da artéria carótida, são sensíveis às alterações na pressão arterial. Durante as elevações na pressão sanguínea, essas células aumentam suas frequências de descarga, transmitindo os impulsos para tronco encefálico (área bulbar). Isso inicia a atividade parassimpática e inibe a resposta simpática, diminuindo a FC e a PA, como mecanismo compensatório em curto prazo (Hinkle \& Cheever, 2020).

Os mecanismos regulatórios da PA incluem o córtex cerebral, o hipotálamo, os centros vasomotores, o SNA, as suprarrenais, os rins, os barorreceptores e algumas vias nervosas especiais, como o nervo de Cyon e o de Hering. O sistema humoral, a cargo dos rins e das suprarrenais, é mediado por várias substancias renina, aldosterona, angiotensina, prostaglandinas, vasopressina, desoxicorticosterona e glicocorticoides (Porto, 2005).

A VFC tem sido indicada como meio não invasivo de avaliação do controle neural do coração. Estudos recentes têm comprovado que a redução da VFC está intimamente relacionada a um maior índice de morbidade e mortalidade cardiovascular (Reis et al., 2002).

O SNA exerce influência no funcionamento de diversos órgãos, aparelhos e sistemas do organismo humano, sendo este essencial para o equilíbrio fisiológico interno, e qualquer alteração que venha a provocar um desequilíbrio provoca uma reação orgânica de forma natural e automática ${ }^{3}$. Desempenha uma função significativa no sistema circulatório e na regulação da PA. Ele inerva as fibras nervosas do coração e os vasos sanguíneos podendo levar a anormalidades no controle da frequência cardíaca (FC) e na dinâmica vascular. A análise da variabilidade da frequência cardíaca (VFC) é uma ferramenta amplamente utilizada para avaliar a regulação autonômica cardíaca (Bassi et al., 2018).

A VFC explica as oscilações da duração dos intervalos entre batimentos cardíacos consecutivos (intervalos RR) e está 
relacionada com as influências do sistema nervoso autônomo sobre o nódulo sinusal, traduzindo a capacidade do coração responder os múltiplos estímulos fisiológicos e ambientais, como, por exemplo, a respiração, o exercício físico, as alterações hemodinâmicas e metabólicas, o ortostatismo, bem como a resposta ao estresse induzido pelas doenças (Bento et al., 2017).

\section{Conclusão}

A partir dos dados desta pesquisa, considera-se que a manobra de Bag squeezing gerou um efeito satisfatório na SatO2, atenuação das FC e respiratória, além da PA, e repercussões positivas prolongadas na modulação autonômica simpática destes pacientes. Sendo assim, esta manobra é indicada em pacientes hipersecretivos, já que se provou ser uma manobra estimulante do sistema nervoso autônomo simpático; em pacientes com hipossaturação e com aumento dos sinais vitais. Com isso, esta manobra pode ser classificada como de remoção de secreção, regulação simpática e ativação de trocas gasosas.

Sugere-se a realização de ensaios clínicos randomizados abordando a temática para que seja possível ter uma melhor noção sobre o efeito dessa técnica, incluindo a avaliação de variáveis hematimétricas, além da aplicação da técnica em outras populações com características distintas, o que possibilitaria uma análise mais detalhada sobre os efeitos do Bag squeezing.

\section{Referências}

Akpınar, E. E., Hoşgün, D., Akpınar, S., Ateş, C., Baha, A., Gülensoy, E. S., \& Ogan, N. (2019). Do N-terminal pro-brain natriuretic peptide levels determine the prognosis of community acquired pneumonia? Jornal Brasileiro de Pneumologia, 45(4). https://doi.org/10.1590/1806-3713/e20180417

Andrade, S. T. (2018). Manobras de hiperinsuflação mecânica e peep zeep: efeitos hemodinâmicos. Universidade Federal de Uberlândia,.

Azeredo, C. A. C. (2002). Fisioterapia respiratória moderna (1 $\left.{ }^{\text {a }}\right)$. Editora Manole.

Bassi, D., Cabiddu, R., Mendes, R. G., Tossini, N., Arakelian, V. M., Caruso, F. C. R., Bonjorno Júnior, J. C., Arena, R., \& Borghi-Silva, A. (2018). Effects of Coexistence Hypertension and Type II Diabetes on Heart Rate Variability and Cardiorespiratory Fitness. Arquivos Brasileiros de Cardiologia, 111(1). https://doi.org/10.5935/abc.20180105

Bento, L., Fonseca-Pinto, R., \& Póvoa, P. (2017). Autonomic nervous system monitoring in intensive care as a prognostic tool. Systematic review. Revista Brasileira de Terapia Intensiva, 29(4). https://doi.org/10.5935/0103-507X.20170072

Berti, J. S. W., Tonon, E., Ronchi, C. F., Berti, H. W., Stefano, L. M. de, Gut, A. L., Padovani, C. R., \& Ferreira, A. L. A. (2012). Hiperinsuflação manual combinada com compressão torácica expiratória para redução do período de internação em UTI em pacientes críticos sob ventilação mecânica. Jornal Brasileiro de Pneumologia, 38(4), 477-486. https://doi.org/10.1590/S1806-37132012000400010

Brasil. (2013). Resolução ${ }^{\circ}$ 466, de 12 de dezembro de 2012. Dispõe sobre diretrizes e normas regulamentadoras de pesquisas envolvendo seres humanos. Diário Oficial [Da] República Federativa Do Brasil, Brasília, DF.

Cambri, L. T. ., Olivera, F. R., \& Gevaerd, M. S. . (2008). Cardiac autonomic modulation in rest and metabolic control in subjects with type 2 diabetes. $H U$ Revista, Juiz de Fora, 34(2), 115-121.

Cambri, L. T., Fronchetti, L., Oliveira, F. R., \& Gevaerd, M. da S. (2008). Variabilidade da frequência cardíaca e controle metabólico. Arq Sanny Pesq Saúde, 1(1), 72-82. https://www.researchgate.net/publication/258046879\%0Ahttp://medcontent.metapress.com/index/A65RM03P4874243N.pdf

Carvalho, M. H. R. de, Carvalho, S. M. R. de, Laurenti, R., \& Payão, S. L. M. (2014). Tendência de mortalidade de idosos por doenças crônicas no município de Marília-SP, Brasil: 1998 a 2000 e 2005 a 2007. Epidemiologia e Serviços de Saúde, 23(2), 347-354. https://doi.org/10.5123/S1679-49742014000200016

Gomes, G. B., \& Gomes, V. R. (2012). Perfil do idoso internado em UTI do interior paulista. Rev. Cienti. Do UNIFAE, 5(2), 41-46.

Gouveia, G. P. de M. (2018). Impacto Do Método Bad Ragaz Na Funcionalidade, No Sistema Respiratório E Nos Biomarcadores De Estresse Oxidativo Em Idosos Diabéticos Tipo 2, Parnaíba-Pi: Ensaio Clínico Randomizado. Universidade Federal do Ceará.

Hinkle, J. L., \& Cheever, K. H. (2020). Brunner \& Suddarth - Tratado de Enfermagem Médico-Cirúrgica (14ª). Guanabara Koogan.

Kanso, S., Romero, D. E., Leite, I. da C., \& Marques, A. (2013). A evitabilidade de óbitos entre idosos em São Paulo, Brasil: análise das principais causas de morte. Cadernos de Saúde Pública, 29(4), 735-748. https://doi.org/10.1590/S0102-311X2013000400011

Lobo, D. M. L., Cavalcante, L. A., \& Mont'Alverne, D. G. B. (2010). Aplicabilidade das técnicas de bag squeezing e manobra zeep em pacientes submetidos à ventilação mecânica. Revista Brasileira de Terapia Intensiva, 22(2), 186-191. https://doi.org/10.1590/S0103-507X2010000200013

Macedo, S. E. C., Menezes, A. M. B., Albernaz, E., Post, P., \& Knorst, M. (2007). Fatores de risco para internação por doença respiratória aguda em crianças 
Research, Society and Development, v. 10, n. 6, e17310615662, 2021

(CC BY 4.0) | ISSN 2525-3409 | DOI: http://dx.doi.org/10.33448/rsd-v10i6.15662

até um ano de idade. Revista de Saúde Pública, 41(3), 351-358. https://doi.org/10.1590/S0034-89102007000300005

Mata, C. E. F. da, Bueno, M. L. de A., Paiva Neto, A., \& Silva Jr, A. (2012). Efeitos das distâncias nadadas e das respostas da frequência cardíaca durante o treinamento de natação na faixa etária de 13 a 17 anos. EFDeportes.Com, Revista Digital, 166.

Meneses, G. S., Leorne, R. O., Gouveia, S. S. V., \& Gouveia, G. P. de M. (2013). Correlação das alterações osteomioarticulares e dor em idosos de Morrinhos(CE ). Revista Brasileira De Ciências Do Envelhecimento Humano, 10(2).

Novoa, P. C. R., \& Novoa, P. C. R. (2014). What changes in Research Ethics in Brazil: Resolution no. 466/12 of the National Health Council. Einstein (São Paulo), 12(1), vii-vix. https://doi.org/10.1590/S1679-45082014ED3077

Paschoal, M., Volanti, V., Pires, C., \& Fernandes, F. (2006). Variabilidade da frequiência cardíaca em diferentes faixas etárias. Revista Brasileira de Fisioterapia, 10(4), 413-419. https://doi.org/10.1590/S1413-35552006000400009

Pina, R. Z., Lapchinsk, L. F., \& Pupulim, J. S. L. (2009). Percepção de pacientes sobre o período de internação em unidade de terapia intensiva. Ciência, Cuidado e Saúde, 7(4). https://doi.org/10.4025/cienccuidsaude.v7i4.6658

Porto, C. C. (2005). Semiologia médica. Guanabara Koogan.

Reis, A. F. dos, Bastos, B. G., Mesquita, E. T., Romêo Fo, L. J. M., \& Nóbrega, A. C. L. da. (2002). Disfunção parassimpática, variabilidade da freqüência cardíaca e estimulação colinérgica após infarto agudo do miocárdico. Arquivos Brasileiros de Cardiologia, 70(3). https://doi.org/10.1590/S0066782X1998000300011

Rossetto, C., Soares, J. V., Brandão, M. L., Rosa, N. G. da, \& Rosset, I. (2019). Causes of hospital admissions and deaths among Brazilian elders between 2005 and 2015. Revista Gaúcha de Enfermagem, 40. https://doi.org/10.1590/1983-1447.2019.20190201 